\title{
Norm based Threshold Selection for Fault Detectors
}

\author{
Rank, Mike Lind; Niemann, Henrik
}

Published in:

American Control Conference, 1998. Proceedings of the 1998

Link to article, DOI:

10.1109/ACC. 1998.702982

Publication date:

1998

Document Version

Publisher's PDF, also known as Version of record

Link back to DTU Orbit

Citation (APA):

Rank, M. L., \& Niemann, H. (1998). Norm based Threshold Selection for Fault Detectors. In American Control Conference, 1998. Proceedings of the 1998 (Vol. 4, pp. 2027-2031). IEEE.

https://doi.org/10.1109/ACC.1998.702982

\section{General rights}

Copyright and moral rights for the publications made accessible in the public portal are retained by the authors and/or other copyright owners and it is a condition of accessing publications that users recognise and abide by the legal requirements associated with these rights.

- Users may download and print one copy of any publication from the public portal for the purpose of private study or research.

- You may not further distribute the material or use it for any profit-making activity or commercial gain

- You may freely distribute the URL identifying the publication in the public portal

If you believe that this document breaches copyright please contact us providing details, and we will remove access to the work immediately and investigate your claim 


\section{Norm based Threshold Selection for Fault Detectors}

\author{
Mike Lind Rank \\ Department of Automation \\ Technical University of Denmark \\ Building 326, DK-2800 Lyngby, Denmark. \\ E-mail: mlr@iau.dtu.dk
}

\author{
Henrik Niemann* \\ Dept. of System Engineering \\ Australian National University \\ Canberra ACT 0200, Australia \\ E-mail: hhn@iau.dtu.dk
}

\begin{abstract}
The design of fault detectors for fault detection and isolation (FDI) in dynamic systems is considered in this paper from a norm based point of view. An analysis of norm based threshold selection is given based on different formulations of FDI problems. Both the nominal FDI problem as well as the uncertain FDI problem will be considered. Based on this analysis, a performance index based on norms of the involved transfer functions is given. The performance index allow also to optimize the structure of the fault detection filter directly.
\end{abstract}

\section{Introduction}

Today there exist various design methods for fault detectors $[6,8]$. Different optimizations techniques as $\mathcal{H}_{\infty}$ and $\mathcal{L}_{1}$ optimization have been applied in e.g. $[1,5,7,9]$. However, the design of a fault detector is only the half part of a full FDI design. The other part is the selection/calculation of a threshold for the fault detector. This second part may be the most important part and often the more difficult. One of the reasons is that there does not exist any systematic way to obtain a threshold value. The threshold selection problem has been considered in a number of papers e.g. $[2,3,4,5]$.

The selection of the threshold is quite closely related to the ability to reject disturbances and model uncertainties and at the same time to be able to get a reasonably detection of fault signals. Based on this fact, different performance indices for the fault detection problem can be found in the literature which reflect the above properties. Examples of these indices can be found in [2] and [9]. The index derived in [2] results in an optimization problem which can not be solved in a systematic way. The reason is that the index include a maximization of

*On leave from Department of Automation, Technical University of Denmark, Building 326, DK-2800 Lyngby, Denmark. Supported by the Danish Technical Research Council under grant no. 96-01557. the smallest gain of a transfer function. The index applied in [9] include only an optimization of the maximal gains of some transfer functions, which can be done in an optimal way. However, the smallest gain from fault signal to estimated fault signal is more important. Since by comparing this gain with the maximal gain from disturbance to estimated fault signal one obtains an indication of how small fault signal that can be estimated. This is precisely what the index derived in [2] do.

The main topic for this paper is to investigate the selection of thresholds and performance indices in connection with FDI. The investigation of threshold selection will be done by using norms on the related transfer functions. The results presented in this paper can be applied in connection with all types of fault detectors. Based on an analysis of the fault detection problem, an index is derived which reflect the design constraint on the transfer functions from disturbance and fault signals to the estimation signals.

\section{FDI Setup}

The FDI design setup will be given in the following. Consider the following system $G$ given by:

$$
\begin{aligned}
& \dot{x}=A x+B_{d} d+B_{f} f \\
& y=C_{y} x+D_{y d} d+D_{y f} f
\end{aligned}
$$

or as transfer functions:

$$
y=\left(\begin{array}{ll}
G_{y f} & G_{y d}
\end{array}\right)\left(\begin{array}{l}
f \\
d
\end{array}\right)
$$

(We shall throughout the paper assume 'compatible' dimensions of vectors and matrices to avoid tedious listing of dimensions.) $d$ is a disturbance signal vector and $f$ is a fault signal vector. It is without loss of generality to consider a system without a control input in connection with FDI design.

In the rest of this paper it will be assumed that the fault signal $f$ and the disturbance signal $d$ are scaled 
such that the norms of these two vectors are unity, i.e.

$$
\|f\| \leq 1, \quad\|d\| \leq 1
$$

and the scaling functions, $S_{f}, S_{d}$, are included in the two transfer functions $G_{y f}$ and $G_{y d}$.

A filter, $F(s)$, is now applied to estimate the fault signal vector $f$ out from the measurement signal vector $y$ i.e. $\hat{f}=F(s) y$. The estimation signal is then given by:

$$
\hat{f}=F(s)\left(G_{y d}(s) d+G_{y f}(s) f\right)
$$

The design problem is to find a fault detector/filter $F(s)$ such that the fault signal $f$ is estimated as good as possible and the noise signal $d$ is rejected at the same time. If an optimization design method is to be applied for the design of $F(s)$, it will in general be more convenient to use the fault detection error given by:

$$
e_{n o m}=f-\hat{f}=f-F(s)\left(G_{y d}(s) d+G_{y f}(s) f\right)
$$

\section{Analysis of the Nominal Case}

The selection of thresholds for FDI filters in the nominal case will be analyzed in this section. Instead of using the estimated fault signal directly as done normally, we will apply the fault estimation error instead. The reason is that we will be able to give guarantee for detection and base our performance index on norms of transfer functions.

Let the smallest gain of $M$ defined as inf $\|x\|=1\|M x\|$ be denoted $\|M\|_{-}$, however, this is not a norm. Based on the the following results we relate the smallest gain and the estimation error, which we later use to setup a performance index. First we take a look at the $\mathcal{H}_{\infty}$ system norm

Lemma 1 Given a transfer function $M \in \mathcal{R} \mathcal{H}_{\infty}$ s.t.

$$
\|(I-M)\|_{\infty} \leq \alpha \leq 1
$$

then

$$
\inf _{\omega \in \mathbf{R}} \sigma_{\min }(M(\omega)) \geq 1-\alpha
$$

This is the case for any induced norm of which above lemma is special case.

Lemma 2 Given a transfer function $M$ in an induced norm space s.t.

$$
\|(I-M)\|:=\sup _{\|x\|=1}\|(I-M) x\| \leq \alpha \leq 1
$$

then

$$
\inf _{\|x\|=1}\|M x\| \geq 1-\alpha
$$

Proof.

$$
\begin{aligned}
& \inf _{\|x\|=1}\|M x\|=\inf _{\|x\|=1}\|I x-(I-M) x\| \geq \\
& \inf _{\|x\|=1}(\|I x\|-\|(I-M) x\|) \\
& \geq 1-\sup _{\|x\|=1}\|(I-M) x\| \geq 1-\alpha
\end{aligned}
$$

Lemma 3 Given transfer functions $W, M$ in an induced norm space s.t.

$$
\inf _{\|x\|=1}\|W x\|=\delta,\|(W-M)\| \leq \alpha \leq \delta
$$

then

$$
\inf _{\|x\|=1}\|M x\| \geq \delta-\alpha
$$

We first assume no scaling of faults is needed, $\|f\| \leq 1$ with $S_{f}=I$. By the lemmas we have a lower bound on the smallest gain based on an induced norm.

As we only are interested in having (or it is only possible to obtain) a small error in a certain frequency region, e.g. low pass or bandpass we introduce a normalized weight $W_{e}(\omega)$ with $\left\|W_{e}\right\| \leq 1$

Let the norms of the two transfer functions $W_{e}(I-$ $\left.F G_{y f}\right)$ and $W_{e} F G_{y d}$ be as follows:

$$
\left\|W_{e}\left(I-F G_{y f}\right)\right\| \leq \alpha,\left\|W_{e} F G_{y d}\right\| \leq \beta
$$

where $\alpha$, maximum estimation error, and $\beta$ must satisfy $\alpha \leq 1$ and $\beta \leq 1$. Note that one is an upper bound and it can always be obtained with $F=0$.

Using Lemma 2 and the triangle-inequality

$$
1-\alpha \leq \inf _{\|x\|=1}\left\|W_{e} F G_{y f} x\right\|,\left\|W_{e} F G_{y f}\right\| \leq 1+\alpha
$$

Using the fault estimation error instead as the fault estimation signal directly, it has been possible to give both an upper bound and more important also a lower bound for the largest and smallest gain of $W_{e} F G_{y f}$.

Based on the norm bounds for $W_{e} F G_{y f}$, we can setup under which condition it is possible to detect a fault signal. Let the threshold value be $T H=\Gamma$.

1. fault signals $f \geq \frac{\Gamma+\beta}{1-\alpha}$ is detected independent of the noise.

2. fault signals $\frac{\beta}{1-\alpha} \leq f \leq \frac{\Gamma+\beta}{1-\alpha}$ may be detected. Noise signals can obstruct a detection.

3. fault signals detected in the range $\frac{\Gamma}{1-\alpha} \leq f \leq \frac{\beta}{1-\alpha}$ may not be fault signals for $\Gamma<\beta$ 
4. fault signals $f \leq \frac{\Gamma}{1-\alpha}$ will not be detected in general, unless the noise signal "helps".

5. no false alarms for $\Gamma \geq \beta$

The above analysis based on norms of the two transfer functions $W_{e} F G_{y f}$ and $W_{e} F G_{y d}$ is somewhat conservative. It is not taken into account that only a limit number of faults is assumed to appear at the same time.

An important thing to note with respect to the above analysis is that the norm of the disturbance transfer function $F G_{y d}, \beta$, is very important in connection with the selection of the threshold value. If we want to avoid false alarms, the threshold value must be selected to $\beta$.

\section{Performance Index}

As pointed out in Section 3, it is quite obvious to select the threshold value equal to or larger then the norm of the weighted transfer function from disturbance to the fault estimation error. An obvious thing to do in the design of the FDI filter is to reduce the norm of this transfer function as much as possible to get as low threshold value as possible. However, this will in general not result in a minimization of the smallest fault signal that can be detected. There is a trade off between good fault detection and good disturbance rejection. This limitation in filtering has been considered in the book of Seron et.al. [10]. Instead, we need to consider a performance index which directly take care of a minimization of the disturbance effect on the estimated fault signal and at the same time maximize the estimated fault signal. Such a performance index has been formulated in e.g. [2] and [4]. The performance index given in [2] is given by:

$$
J=2 \inf \frac{\left\|F G_{y d}\right\|}{\left\|F G_{y f}\right\|_{-}}
$$

The performance index in (6) gives the smallest fault signal that is guaranteed to be detected. A design method which will minimize the index $\mathrm{J}$ in (6) need to be applied. However, the optimization of the performance index is difficult as pointed out in [2], because the denominator is not a norm.

\subsection{Index based on induced norms}

Motivated by section 3 we introduce the following index:

$$
\|f\| \geq \eta=2 \inf _{F} \frac{\left\|W_{e} F G_{y d}\right\|}{1-\left\|W_{e}\left(I-F G_{y f}\right)\right\|}
$$

where the normalized weight $W_{e}(\omega)$ with $\left\|W_{e}\right\| \leq 1$ reflects the frequency region of interest, eg. low pass or bandpass. Besides the weight must be small around frequencies where $G_{y f}$ have RHP zeros and likewise for LHP zeros else $\eta$ will be very conservative. When $S_{f} \neq I$ replace 1 with $\delta=\inf _{\|x\|=1}\left\|S_{f} x\right\|$ and $I$ with $S_{f}$ in $(7)$.

Faults $\|f\| \geq \eta$ are guaranteed to be detected, however, $\eta \geq J$, the bound may be conservative, but it is here important to note that the index in (7) only include norms of transfer functions. This will make an optimization of the index much more simple than an optimization of the index given by (6).

Further, by using the index in (7), we do not only have the possibility to optimize the size of the fault signal that is guaranteed to be detected, it is also possible to give a structure for the FDI filter in connection with the optimization. This is important to improve the performance of the FDI filter. This can be done by changing the equation for the fault estimation error given by (3) to,

$$
e_{n o m}=\left(V-F(s) G_{y f}(s)\right) f-F(s) G_{y d} d
$$

where $V$ is a constant matrix. With the selection of $V$, it is possible to put structure in the optimization of the FDI filter. The motivation for using this more general equation for the estimation error is that we get, in a very simple way, a possibility to include structure in the filter. It is not necessarily optimal to design the filter such that it obtain a diagonal structure. From a performance point of view, it can be better to design a filter with a triangle structure [10]. It should be noted, that it is also possible to let $V$ include dynamics.

Theorem 4 Let the fault estimation error be given by (8), where the constant matrix $V$ satisfied $\inf _{\|x\|=1}\|V x\|=\delta$. Further, it is assumed that $\| W_{e}(V-$ $\left.F G_{y f}\right) \| \leq \delta$, where the weight matrix satisfies $\left\|W_{e}\right\| \leq$ 1. Then the performance index which need to be optimized is given by

$$
\|f\| \geq \eta=2 \inf _{F} \frac{\left\|W_{e} F G_{y d^{\prime}}\right\|}{\delta-\left\|W_{e}\left(V-F G_{y f}\right)\right\|}
$$

Proof. Theorem 4 follows directly by using the result in Lemma 2 at the equation for the fault estimation error in (8).

In the SISO case, where $\left|W_{e}\right| \approx 1$, we get directly that it is required that $\left|G_{y f}\right|>2\left|G_{y d}\right|$ to get guarantee for any fault detection, i.e. $\eta<1$. Another case is when the disturbance is measurement noise, i.e. $G_{y d}=k=$ constant. To obtain fault detection, it is required that $\left|G_{y f}\right|>2 k$ in the frequency range where fault detection is wanted. 


\subsection{Index with two system norms}

The performance index (7) makes sense whenever maximal estimation error is bounded by an induced norm hence in general it makes sense to consider

$$
\eta=2 \inf _{F} \frac{\left\|W_{e} F G_{y d}\right\|_{a}}{1-\left\|W_{e}\left(I-F G_{y f}\right)\right\|}
$$

where $\|\cdot\|_{a}$ is any system norm, with $\left\|W_{e}\right\| \leq 1$. Hence the disturbance attenuation may be with respect to white noise, i.e. $\mathcal{H}_{2}$, whereas the estimation error minimization may be over energy or amplitude induced norm.

\subsection{Index for a reduced number of si- multaneously faults}

The performance indices given above are all based on the possibility that all fault signals can appear at the same time. However, in practice, there will in general be an assumption on the number of faults that will/can appear at the same time. This mean that the performance index given in Theorem 4 might be conservative.

Consider a FDI problem where $m$ simultaneously faults can appear. Assumed that only $p,(p<m)$ faults can appear at the same time. To rewrite the index, let the transfer function $W_{e}\left(V-F G_{y f}\right)$ be partitioned as:

$$
W_{e}\left(V-F G_{y f}\right)=\left[g_{w 1}, \ldots, g_{w m}\right]
$$

The worst case gain of $W_{e}\left(V-F G_{y f}\right)$ when only $p$ fault signals can appear at the same time is then given by:

$$
\max \left\{\sum_{i_{1}=1}^{i_{1}=m} \ldots \sum_{i_{p}=p}^{i_{p}=m}\left\|g_{w i_{1}} \ldots g_{w i_{p}}\right\|\right\}, i_{1} \neq \ldots \neq i_{p}
$$

The resulting index may be solved by stacking the problem. An important case is when one fault may appear,

$$
\|f\| \geq \eta=2 \inf _{F} \frac{\left\|W_{e} F G_{y d}\right\|}{\delta-\max \left\{\sum_{i_{1}=1}^{i_{1}=m}\left\|g_{w i_{1}}\right\|\right\}}
$$

\section{The Uncertain FDI Setup}

The nominal FDI setup described in Section 2 will be extended in this section to uncertain systems. Consider the following uncertain system $G_{u n c}$ given by:

$$
\left(\begin{array}{c}
z \\
y
\end{array}\right)=\left(\begin{array}{ccc}
G_{z w} & G_{z f} & G_{z d} \\
G_{y w} & G_{y f} & G_{y d}
\end{array}\right)\left(\begin{array}{c}
w \\
f \\
d
\end{array}\right)
$$

where $z$ and $y$ are the external output signal and the measurement output signal, respectively. The inputs signals are the external input signal $w$, the fault signal $f$ and the disturbance input signal $d$.

The external input signal $w$ and the external output signal $z$ is closed through the uncertain perturbation block $\Delta(s)$, i.e. $w=\Delta(s) z$. Assume that the perturbation block $\Delta(s)$ is scaled s.t. $\|\Delta\| \leq 1, \forall \omega$ and the scaling function is included in $G_{u n c}$.

Based on the results in Section 3, we consider again the transfer function for the fault estimation error $e_{u n c}$. In the uncertain case, the estimation error is given by:

$$
e_{u n c}=f-\hat{f}=f-F(s)\left(G_{y w} w+G_{y f}(s) f+G_{y d}(s) d\right)
$$

in the open loop case. When this loop is closed, Figure 1, the fault estimation error take the following form by using (3) and $S_{\Delta}=\left(I-G_{z w} \Delta\right)^{-1}$,

$$
e_{u n c}=e_{n o m}-F(s) G_{y w} \Delta S_{\Delta}\left(G_{z f} f+G_{z d} d\right)
$$

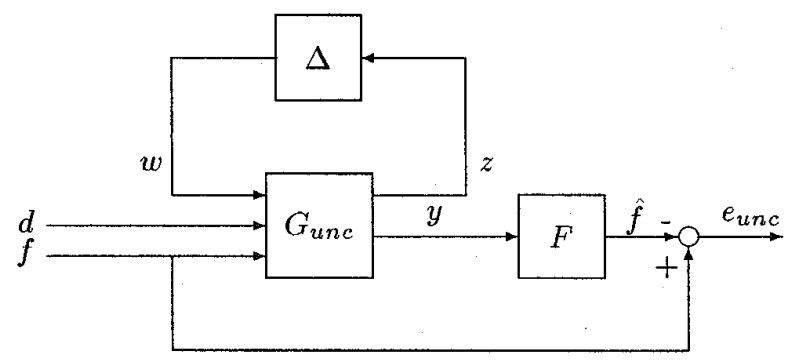

Figure 1: Block diagram for the fault detection error

\section{The Uncertain FDI case}

Based on the results derived in Section 3 and 4 for the nominal case, equivalent results will be given in this section for the open-loop uncertain case. One of the key results from Section 3 was that if the fault detection error is applied, the performance index given by (7) is only based on norms.

Let the norm of the two transfer functions from (13) be given by:

$$
\begin{gathered}
\left\|W_{e}\left(I-F\left(G_{y w} \Delta S_{\Delta} G_{z f}-G_{y f}\right)\right)\right\| \leq \alpha \\
\left\|W_{e}\left(F\left(G_{y w} \Delta S_{\Delta} G_{z d}+G_{y d}\right)\right)\right\| \leq \beta
\end{gathered}
$$

where $W_{e}$ is a weight function with $\left\|W_{e}\right\| \leq 1$.

Based on the norms of these two transfer functions, we can directly give an equation for the threshold value 
which need to be optimized. In the case when we do not want any false alarms, select $\Gamma \geq \beta$ and the smallest fault signal is guarantee to be detected is given by the performance index:

$$
\eta=2 \inf _{F} \frac{\left\|W_{e}\left(F\left(G_{y w} \Delta S_{\Delta} G_{z d}+G_{y d}\right)\right)\right\|}{1-\left\|W_{e} F\left(G_{y w} \Delta S_{\Delta} G_{z f}+G_{y f}\right)\right\|}
$$

The optimization problem in the uncertain case is therefore exactly the same as in the nominal case given by (7). In connection with this optimization problem, it should be mentioned that the problem of selection of thresholds for the uncertain FDI problem has also been considered in [2]. As mention before, the results derived in [2] are based on the based on the fault estimation signal directly. Further, only systems with open loop uncertainties are considered. Instead of using the description by feeding back the uncertain block, it is instead assumed that the input to the uncertain block is bounded. This will in general give a too simple description of the uncertain part of the system.

\section{Conclusion}

An analysis of the threshold selection problem has been given in this paper for both nominal as well as for uncertain systems. The main contribution in this paper is the formulation of the fault detection and isolation problem in terms of induced norms of transfer functions. This has been possible by using the fault estimation error instead of using the estimated fault signal directly. A new performance index for the optimization of FDI filters has been given in terms of norms of transfer functions for both nominal as well as for uncertain systems. By using this performance index, it is possible to use design methods from robust and optimal control for the optimization of the index in a systematic way.

Further, it has been shown how it is possible to include structure in the design of the FDI filter directly without any modification of the applied optimization method, which is not possible directly with other known performance indices for FDI design. It is important to have the possibility to apply other structures than a diagonal structure for the filter, when there is a trade off between estimation and disturbance rejection.

Applying the new performance index, we will obtain an optimal detection filter with respect to detection the smallest possible fault signal. In contrary using to an optimization method directly on the FDI problem.

In this paper we have only discussed the selection of the threshold equal to or larger than the norm of the trans- fer function from disturbance to estimation error. This selection will avoid any false alarms. If the threshold is reduced, false alarms may be obtained. An analysis need to be done in every single case to give the level of the threshold if a number of false alarms can be accepted. Such a reduction of the threshold level will not change the performance index.

\section{References}

[1] H. Ajbar and J.C. Kantor. An $\ell_{\infty}$ approach to robust control and fault detection. In Proceedings of the American Control Conference, pages 3197-3201, San Francisco, CA, USA, 1993.

[2] X. Ding and L. Guo. Observer based optimal fault detector. In Proceedings of the 13th IFAC World Congress, volume N, pages 187-192, San Francisco, CA, USA, 1996.

[3] X. Ding, L. Guo, and P.M. Frank. A frequency domain approach to fault detection of uncertain dynamic systems. In Proceedings of the 32nd Conference on Decision and Control, pages 1722-1727, San Antonio, TX, 1993.

[4] A. Emami-Naeini, M.M. Akhter, and S.M. Rock. Effect of model uncertainty on failure detection: The threshold selector. IEEE Transactions on Automatic Control, 33:1106-1115, 1988.

[5] Y.E. Faitakis and J.C. Kantork. Residual generation and fault detection for discrete-time systems using $\ell_{\infty}$ technique. International Journal of Control, pages 155 $174,1996$.

[6] P.M. Frank. Analytical and qualitative model-based fault diagnosis - A survey and some new results. European Journal of Control, 2:6-28, 1996.

[7] H.H. Niemann and J. Stoustrup. Filter design for failure detection and isolation in the presence of modeling errors and disturbances. In Proceedings of the 35th IEEE Conference on Decision and Control, pages 1155-1160, Kobe, Japan, 1996.

[8] R. Patton, P. Frank, and R. Clark. Fault diagnosis in dynamic systems - Theory and application. Prentice Hall, 1989.

[9] Z. Qiu and J. Gertler. Robust FDI systems and $\mathcal{H}_{\infty}$ optimization. In Proceedings of the 32nd Conference on Decision and Control, pages 1710-1715, San Antonio, Texas, USA, 1993.

[10] M.M. Seron, J. H. Braslavsky, and G.C. Goodwin. Fundamental limitations in filtering and control. SpringerVerlag, 1997. 